\title{
Narrow band imaging survey of dark clouds
}

\author{
Movsessian T.A. ${ }^{* 1}$, Magakyan T.Yu. ${ }^{\dagger 1}$, Dodonov S.N. ${ }^{\ddagger 2}$, and Andreasyan H.R..$^{\S 1}$ \\ ${ }^{1}$ Byurakan Astrophysical Observatory, Aragatsotn reg., Byurakan, 0213 Armenia \\ ${ }^{2}$ Spetial Astrophysical Observatory, N.Arkhyz, Karachaevo-Cherkesia, 369167 Russia
}

\begin{abstract}
We present new results obtained in frames of the Byurakan Narrow Band Imaging Survey (BNBIS). Observations were carried out with upgraded $1 \mathrm{~m}$ Schmidt telescope of Byurakan Observatory. Main task of this survey is to search and to study the new Herbig-Haro objects and collimated outflows using narrow-band $\mathrm{H} \alpha$ and [S II] images of some fields in dark clouds of Galaxy. And, because, Herbig-Haro objects are the main indicators of active starformation processes to fined regions where going on active starformation processes. Main targets of this survey are $\mathrm{R}$ associations, young stellar objects associated with compact reflection nebulae, but here we will mainly focused on deeply embedded infrared sources in molecular clouds.

During this survey we plan to significantly expand the list of HH objects by using the high quantum efficiency of the system and the telescopes high focal ratio (F/2), which allows detecting low surface brightness objects as well as large field of view of Schmidt telescope.
\end{abstract}

Keywords: Starformation, Herbig-Haro objects, jets and outflows

\section{Introduction}

Herbig-Aro objects are formed by the interaction of collimated outflows from young stellar objects with the circumstellar medium and, in some cases, represents the brightest parts of optical jets. It is considered proven that the presence of Herbig-Haro objects is one of the main signs of an active star formation process in dark clouds (Reipurth \& Bally, 2001). In most cases the sources of Herbig-Haro objects are stars which are bright in the infrared region of the spectrum, and only a small part of them are optically bright stars, which are mainly classical stars of the Tau class. Discovering of new HH objects are important as for the further studies of the phenomenon of directed outflows from young stars in various evolutionary stages, as well as for the searches for new star forming regions and groups.

The presence of deeply embedded sources with an infrared excess, characteristic of young stellar objects, can also be considered as a sign of star formation. Such sources are divided into three classes according to their infrared excess calculated by their spectral indexes. Currently, three classes have been identified, Class I - considered candidates for protostars, class II - T Tau stars and class III WTTs-type stars. In addition to these three classes, Class 0 has been proposed as evolutionary precursors of Class I protostars (Andre, Ward-Thompson, \& Barsony, 1993, Andre \& Montmerle, 1994). The work of (Bontemps et al., 1996) and (Saraceno et al., 1996). However, some Class I objects that are observed at a high inclination may look 'Class 0-like' due to the high optical thickness associated with absorption in a dense circumstellar disk (Masunaga \& Inutsuka, 2000). This circumstance further complicates the distinction between Class 0 and Class I objects, however, there are sources associated with reflection nebulae, which helps clarify the nature of the source. The presence of such nebulae indicates that the optical radiation of the source is obscured not only by a dark cloud, since a reflective nebula is visible, but also by a dense circumstellar disk.

Taking this into account, we have included dark clouds with bright infrared sources and especially those associated with compact reflective nebulae in the list of studied areas.

\footnotetext{
*tigmov@bao.sci.am, Corresponding author

†tigmag@sci.am

${ }^{\ddagger}$ dodo@sao.ru

${ }^{\S}$ D.D@university.edu
} 


\section{Observations}

The images were obtained on the nights of Feb. 3-4 2019 with 1m Schmidt telescope of Byurakan observatory, which was upgraded during 2013-2015 and equipped with CCD detector. Reworked $4 \mathrm{~K} \times 4 \mathrm{~K}$ Apogee (USA) liquid-cooled CCD camera was used as a detector with a pixel size of 0.868 " and field of view of about 1 square degree (Dodonov et al., 2017).

Narrow-band filters centered on $6560 \AA$ and $6760 \AA$, both with a FWHM of $100 \AA$, were used to obtain $\mathrm{H} \alpha$ and [S II] images, respectively. A midband filter, centered on $7500 \AA$ with a FWHM of $250 \AA$, was used for the continuum imaging.

A dithered set of 5 min exposures was obtained in each filter. Ususal effective exposure times were about 3600-7200 sec in $\mathrm{H} \alpha$ and [S II] filters. Images were reduced in the standard manner using IDL package developed by one of authors (SND), which includes bias subtraction, cosmic ray removal, and flat fielding using "super flat-field", constructed by several images.

\section{Results}

\subsection{Mon R1-first success}

R1 association located not far from Mon OB1 (see Dahm (2003), for a detailed review) but unlike of it has not been investigated in sufficient detail. In frames of BNBIS in the Mon R1 were discovered several new HH objects as well as two collimated outflow systems, one form which belongs to giant HH outflows with size of $1.6 \mathrm{pc}$. Detailed description of newly discovered HH objects and outflow systems can be found in Movsessian, Magakian, \& Dodonov (2021). Narrow band image, which is covering one square degree field of Mon R1 region with newly discovered HH objects, is shown on the Fig. 1 in mentioned paper.

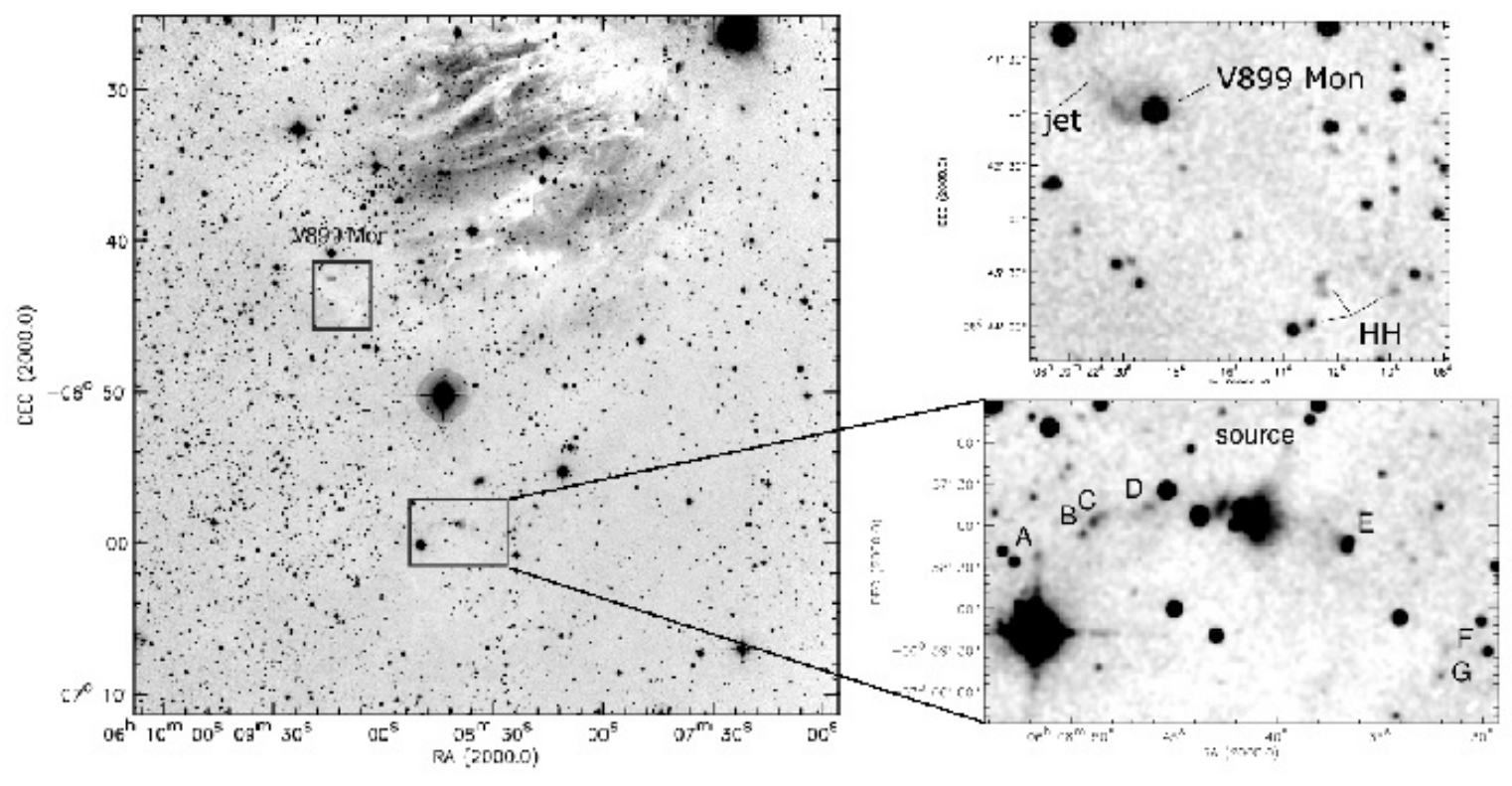

Figure 1. Whole observed field of Mon R2 south Mon in $\mathrm{H} \alpha+[\mathrm{S} \mathrm{II}]$ emission, where the areas including newly discovered $\mathrm{HH}$ objects and outflows, are shown by rectangles (left panel). V899 Mon with associated HH objects and narrow jet (right top) and curved HH outflow system from 2MASS 06084223-0657385 infrared source associated with bipolar reflection nebula (right bottom).

\subsection{RNO 66 and V899 Mon field}

This field located about one degree south from Mon R2 association. Mon R2 association is a well studied region of star formation which contains early-type stars (Racine, 1968), molecular outflows (Meyers-Rice \& Lada, 1991), an embedded HII region (Downes et al., 1975) and clusters of infrared sources (Thronson et al., 1980). However there is little research on the search for HH objects, and were found some on the Eastern 
side of Mon R2 (Carballo \& Eiroa, 1993). Our attention was drawn to the area South of the Central part of Mon R2, where several nebulous objects and an eruptive star V899 Mon are located.

A $\mathrm{H} \alpha+[\mathrm{S} \mathrm{II}]$ image, which is covering one square degree field of the Mon R2 region, is shown on Fig.1 (left panel). The zones with newly discovered $\mathrm{HH}$ objects and $\mathrm{HH}$ flows are marked by rectangles.

\subsubsection{V899 Mon}

A possible FUor-type eruption of V899 Mon(IRAS 06068-0641) located near the Monoceros R2 region (d $\approx 900$ pc; (Lombardi, Alves, \& Lada, 2011) was first discovered by the Catalina Real-time Transient Survey (CRTS) and reported by Wils et al. (2009). They announced the source as a FUor candidate based on the constant brightening it has been undergoing since 2005. The spectrum published by Wils et al. (2009) showed strong $\mathrm{H} \alpha$ and CaII IR triplet lines, which identify the outbursting source as a YSO.

Further optical and NIR spectroscopy of V899 Mon confirms it to be a member of the FUors/EXors family of outbursts. Photometrically and spectroscopically V899 Mon's properties lie between EXors and classical FUors. But it is probably more similar to EXors than classical FUors.

On the distance of about 2,5 arcmin in south-west from V899 Mon four HH knots were discovered which can probably associated with V899 Mon, besides narrow jet was detected in [S II] by the axis of the reflection nebula associated with V899 Mon Fig.1 (right top panel). All data suggest that V899 Mon is a source of collimated and possibly bipolar outflow and $\mathrm{HH}$ objects can represents counter flow from it. However, the newly found $\mathrm{HH}$ objects could be result of activity of other infrared sources in this area.

\subsubsection{Curved HH flow}

Abut 20 arcmin south-west from V899 Mon were revealed several HH knots near the 2MASS 060842230657385 infrared source associated with bipolar reflection nebula. HH objects lies on a parabolic curve at the apex of which the infrared source is located Fig.1 (right bottom panel). Therefore, it can be argued that we are dealing with a bipolar outflow with an unusual arc-shaped structure. Such a morphology is typical for so named irradiated jets or outflows from the sources with high proper motion. We incline to the second scenario, because irradiated outflows represents very thin emission structures without prominent knotty structures (Bally \& Reipurth, 2001). Taking this scenario into account we estimated the proper motion value of the source which is about $50 \mathrm{~km} \mathrm{~s}^{-1}$, which is quite acceptable. Estimated total length of this bipolar outflow will be about $1.5 \mathrm{pc}$ for the distance of $900 \mathrm{pc}$. This outflow system represents giant or so called parsec scale HH flow as well as HH 1196 in Mon R1.

\subsection{IRAS 06212-1049}

The infrared source IRAS 06212-1049 is associated with a cone-shaped reflective nebula, the axis of which is oriented to the southeast. The deep images of the PanSTARRS survey show a faint nebula in the opposite direction from the source, which indicates the bipolar morphology of the nebula. The object is located near the dark cloud LDN 1652 which is located at a distance of 830 pc. It can be assumed that IRAS 06212-1049 is located in this group and, therefore, its distance can be considered the same. On the images obtained in the $\mathrm{H} \alpha$ and $[S \mathrm{II}]$ emission lines were detected $\mathrm{HH}$ knots, which are directed along the axis of the reflection nebula Fig.2 and, obviously, represent a collimated optical flow. If we take a distance of about $800 \mathrm{pc}$, the total length of the outflow will be about $0.9 \mathrm{pc}$.

A detailed analysis of the entire field made it possible to detect two more Herbig-Aro objects, which are shown in Fig.2. The first object is an isolated emission knot near which there is no any infrared source that could be its source. The second, a faint somewhat extended object with a length of about 6", is located near the infrared source IRAS 06216-1044 and is barely visible on the red maps of DSS2. The distance of this object from IRAS 06216-1044, which can be its source, is about 12" or 0.05 pc.

\subsection{New HH objects near IRAS sources}

As was already mentioned we choose some infrared sources as a targets for narrow band survey. All these sources have considerable infrared excess characteristic for young stellar objects. Some of targets are associated with small reflection nebulae with characteristic cone shape. On the Fig. 3 some examples of such a sources are presented where $\mathrm{HH}$ objects are shown by arrows. 

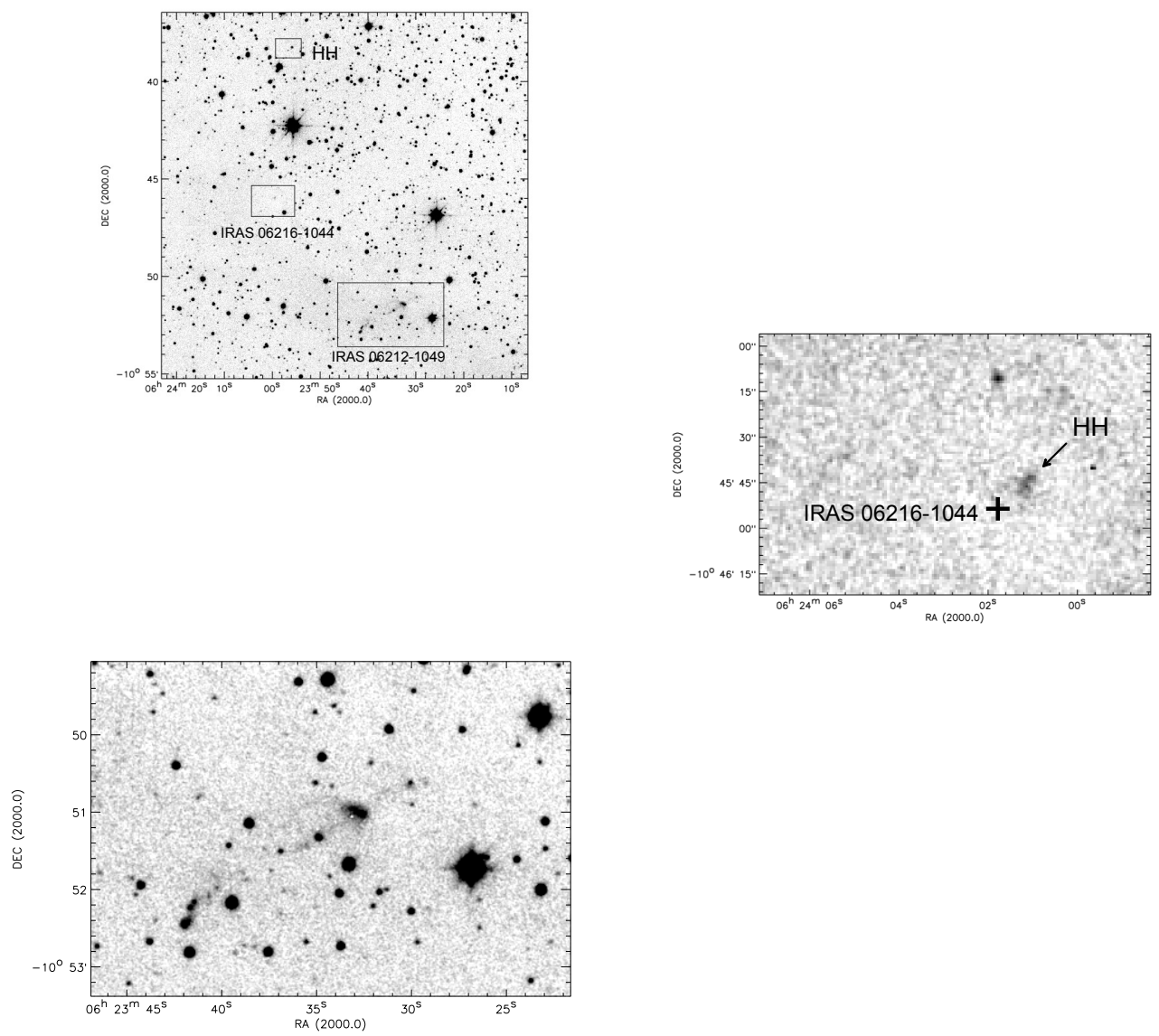

Figure 2. Whole observed field of IRAS 06212-1049 in $\mathrm{H} \alpha+[\mathrm{S}$ II $]$ emission. The areas including newly discovered $\mathrm{HH}$ objects and outflows, are shown by rectangles (left panel). $\mathrm{H} \alpha+[\mathrm{S}$ II] and $[\mathrm{S}$ II] image of IRAS 06212-1049 (bottom panel)and IRAS 06216-1044 (left panel).

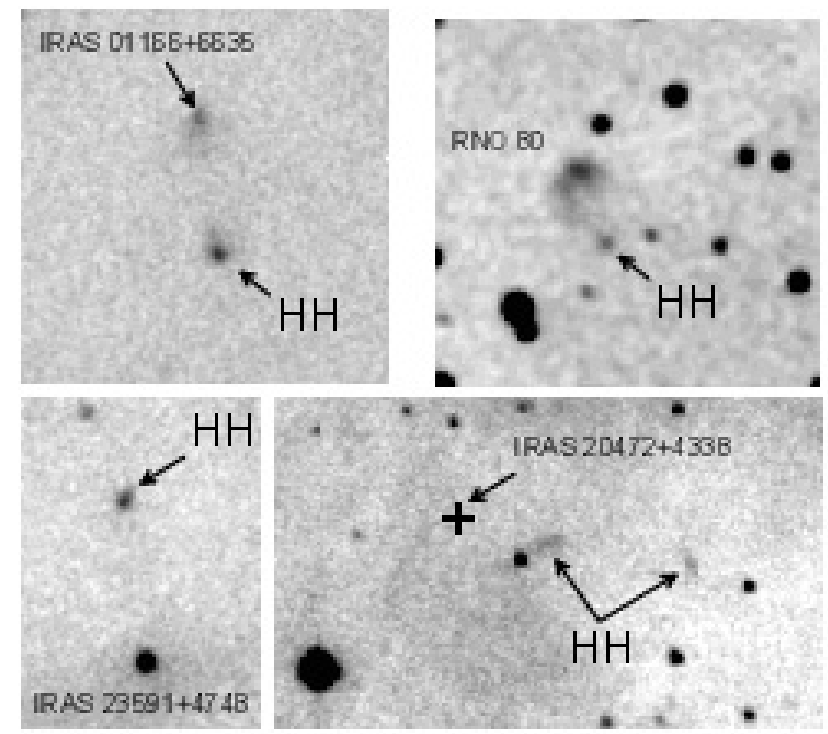

Figure 3. Infrared sources associated with Herbig-Haro objects 


\subsection{Conclusion}

Since the beginning of 2019, when this survey began, more than 100 new HH objects and outflow systems have been found. Among them should be mention several giant outflow systems, narrow jets as well as curved HH flow.

Besides, several new HH objects were discovered near IRAS sources. Analysis of SED's these sources reveal its classes, which are mainly belongs to class I young stellar objects, but some of them have properties of class 0. Taking in account association of those objects with compact reflection nebulae, we incline to consider them as class I sources, which are surrounded by dense disc and oriented edge on.

In addition to all the results presented above, this work demonstrates that the 1-m Schmidt telescope of Byurakan Observatory, which was used several decades ago for well-known surveys of active galaxies such as the First Byurakan Survey and Second Byurakan Survey, can still lead to important discoveries.

\section{References}

Andre P., Ward-Thompson D., Barsony M., 1993, ApJ, 406, 122. doi:10.1086/172425

Andre P., Montmerle T., 1994, ApJ, 420, 837. doi:10.1086/173608

Bally J., Reipurth B., 2001, ApJ, 546, 299. doi:10.1086/318258

Bhadari N. K., Dewangan L. K., Pirogov L. E., Ojha D. K., 2020, ApJ, 899, 167. doi:10.3847/15384357/aba2c6

Bontemps S., Andre P., Terebey S., Cabrit S., 1996, A\&A, 311, 858

Carballo R., Eiroa C., 1993, ASSL, 213. doi:10.1007/978-94-011-1924-5 7

Dahm S.E, 2008, in Reipurth B., ed., Handbook of Star Forming Regions, Vol. I, Astron. Soc. Pac., p. 966

Lombardi M., Alves J., Lada C. J., 2011, A\&A, 535, A16. doi:10.1051/0004-6361/201116915

Dodonov S.N., Kotov S.S., Movsesyan T.A., Gevorkyan M. 2017, Astrophysical Bulletin, 72, 473

Downes D., Winnberg A., Goss W. M., Johansson L. E. B., 1975, AA, 44, 243

Masunaga H., Inutsuka S.-. ichiro ., 2000, ApJ, 531, 350. doi:10.1086/308439

Meyers-Rice B. A., Lada C. J., 1991, ApJ, 368, 445. doi:10.1086/169709

Movsessian T. A., Magakian T. Y., Dodonov S. N., 2021, MNRAS, 500, 2440. doi:10.1093/mnras/staa3302

Reipurth B., Bally J., 2001, ARA\&A, 39, 403. doi:10.1146/annurev.astro.39.1.403

Racine R., 1968, AJ, 73, 233

Saraceno P., Andre P., Ceccarelli C., Griffin M., Molinari S., 1996, A\&A, 309, 827

Thronson H. A., Gatley I., Harvey P. M., Sellgren K., Werner M. W., 1980, ApJ, 237, 66. doi:10.1086/157844

Wils P., Greaves J., Drake A. J., Catelan M., 2009, CBET, 2033 